\title{
Titin-Actin Interaction: PEVK-Actin-Based Viscosity in a Large Animal
}

\section{Charles S. Chung, ${ }^{1}$ Julius Bogomolovas, ${ }^{2}$ Alexander Gasch, ${ }^{2}$ Carlos G. Hidalgo, ${ }^{1}$ Siegfried Labeit, ${ }^{2}$ and Henk L. Granzier ${ }^{1}$}

\author{
${ }^{1}$ Molecular Cardiovascular Research Program, Sarver Heart Center, Department of Physiology, \\ University of Arizona, Tucson, AZ 85724, USA \\ ${ }^{2}$ Department of Integrative Pathophysiology, Universitätsmedizin Mannheim, University of Heidelberg, \\ Theodor-Kutzer-Ufer 1-3, 68167 Mannheim, Germany
}

Correspondence should be addressed to Henk L. Granzier, granzier@email.arizona.edu

Received 23 July 2011; Accepted 1 September 2011

Academic Editor: Guy Benian

Copyright () 2011 Charles S. Chung et al. This is an open access article distributed under the Creative Commons Attribution License, which permits unrestricted use, distribution, and reproduction in any medium, provided the original work is properly cited.

\begin{abstract}
Titin exhibits an interaction between its PEVK segment and the actin filament resulting in viscosity, a speed dependent resistive force, which significantly influences diastolic filling in mice. While diastolic disease is clinically pervasive, humans express a more compliant titin (N2BA:N2B ratio $\sim 0.5-1.0$ ) than mice (N2BA:N2B ratio $\sim 0.2$ ). To examine PEVK-actin based viscosity in compliant titin-tissues, we used pig cardiac tissue that expresses titin isoforms similar to that in humans. Stretch-hold experiments were performed at speeds from 0.1 to 10 lengths/s from slack sarcomere lengths (SL) to SL of $2.15 \mu \mathrm{m}$. Viscosity was calculated from the slope of stress-relaxation vs stretch speed. Recombinant PEVK was added to compete off native interactions and this found to reduce the slope by $35 \%$, suggesting that PEVK-actin interactions are a strong contributor of viscosity. Frequency sweeps were performed at frequencies of $0.1-400 \mathrm{~Hz}$ and recombinant protein reduced viscous moduli by $40 \%$ at $2.15 \mu \mathrm{m}$ and by $50 \%$ at $2.25 \mu \mathrm{m}$, suggesting a SL-dependent nature of viscosity that might prevent SL "overshoot" at long diastolic SLs. This study is the first to show that viscosity is present at physiologic speeds in the pig and supports the physiologic relevance of PEVK-actin interactions in humans in both health and disease.
\end{abstract}

\section{Introduction}

Diastolic (dys)function is of major clinical importance and is characterized by both elastic [1-4] and viscous properties [5-7]. The giant elastic protein titin (also known as connectin $[8,9]$ ) is well known to be related to the elasticity of cardiac muscle impacting the end diastolic pressure volume relationship (for current reviews see [1012]). These elastic properties arise from the three extensible segments localized in the I-band of the sarcomere: the tandem Ig segments, the proline-glutamic acid-lysine-valine rich PEVK element, and the N2B unique sequence. Viscosity is a speed-dependent resistive property of all biological tissues [13]. Viscosity in cardiac tissues impacts diastolic filling by slowing ventricular pressure relaxation and causing a delayed relaxation phenotype during early filling $[2,5,6]$.
The Ig domains that make up the tandem Ig segments of titin's spring region are a possible intrinsic source of viscosity. Individual Ig domains were originally thought to unfold during stretch and refold during release causing a viscosity $[14,15]$, but single molecule and simulation studies suggest that the domain unfolding is unlikely to be a prominent process under physiologic conditions [1620]. Interactions between elements of the extensible I-band of titin and other structures in the sarcomere are another possible source of viscosity. The interaction between titin and actin was hypothesized due to evidence of a viscoelastic interaction in the sarcomere [21], close proximity of the two filament types, and in vitro evidence of titin binding to actin [22-25]. Yamasaki et al. first demonstrated by cosedimentation assays that the PEVK region, but not the N2B element or Ig domains, interacted with actin [13]; 
Kulke et al.'s F-actin cosedimentation assays supported this finding [26]. Both studies showed that F-actin sliding in the in vitro motility assays was slowed by the addition of recombinant PEVK protein to the assay buffer suggestive that the PEVK adds a viscous force that slows filament sliding. Mechanically, Yamasaki found a reduction in the stressresponse of rat cardiac myofibers using exogenously added recombinant PEVK proteins [13]. Kulke utilized gelsolin extraction of thin filaments to abolish native actin-PEVK interaction in myofibrils and found that this reduced viscous stress relaxation [26] (although such extraction potentially releases near-Z-disk titin that is normally firmly bound to actin [27]). Both studies confirmed in the fiber/fibril that a viscous response was due to PEVK-actin interactions. The development of a PEVK KO mouse model [28] provided a new tool to study PEVK-actin interactions and its effect on viscosity. Utilizing skinned cells and fibers from this $\mathrm{KO}$ mouse, a 50\% reduction was found in the viscosity in stressrelaxation and viscous moduli in sinusoidal experiments using protocols identical to the ones used in this study [29]. Recently, using a PEVK KO mouse, we quantified the contribution of PEVK-actin interactions to viscosity in an ex vivo and in vivo whole heart preparation, and we showed a key physiologic consequence that PEVK-actin interactions control $30 \%$ of viscosity during early rapid filling [29].

Cardiac muscle of large mammals, including human, coexpresses both the stiffer N2B isoform and more compliant N2BA isoform unlike that of small rodents that is dominated by expression of the N2B cardiac isoform $[10,12]$. The PEVK element of the N2B isoform contains 7 exons with 5 PPAK (proline-rich) motifs that are negatively charged and involved in actin binding $[28,30]$. The PEVK region of the cardiac N2BA isoform contains all of the elements expressed in the N2B isoform and additional PEVK exons, including additional PPAK repeats as well as two PolyE (glutamic acid rich) motifs [30]. The PolyE motifs appear to have a stronger binding affinity to actin than the PPAK motifs [31]. Because of these changes in PEVK expression and the expression of additional Ig domains in N2BA dominant tissues [12], direct extrapolations from the findings in small animals to humans are difficult. Thus, while characterization of the PEVK-actin interaction shows physiologic relevance in small animals, actual application in large mammals including humans remains unclear. To study viscosity in tissues containing a high $\mathrm{N} 2 \mathrm{BA}: \mathrm{N} 2 \mathrm{~B}$ ratio, we utilized tissues from the pig. Viscous properties were probed using both stretch hold and frequency sweep perturbations to quantify viscosity and we utilized a competition assay with recombinant PEVK proteins to disrupt the native PEVK-actin interactions.

\section{Methods}

2.1. Porcine-Skinned Fiber Preparation and Mechanics. Porcine LV tissue was obtained as previously described [32]. Animal experiments were approved by the University of Arizona Institutional Animal Care and Use Committee and followed NIH guidelines for "Using Animals in Intramural Research." Briefly, the heart was rapidly obtained from the pig $<5$ minutes after sacrifice, washed with a calcium-free
HEPES-buffered solution ([in mM] 10 HEPES; $133.5 \mathrm{NaCl}$, $5 \mathrm{KCl}, 1.2 \mathrm{NaH}_{2} \mathrm{PO}_{4} ; 1.2 \mathrm{MgSO}_{4}, 11$ glucose, $4 \mathrm{Na}$-Pyruvate with $2.5 \mathrm{U} / \mathrm{L}$ insulin, $\mathrm{pH} 7.44$ ), dissected into small strips, and placed in a skinning solution composed of relaxing solution ([in $\mathrm{mM}$ ]: $10 \mathrm{BES}$, $10 \mathrm{EGTA}, 6.56 \mathrm{MgCl}_{2}, 5.88 \mathrm{Na}$-ATP, 1 DTT, 46.35 potassium-propionate, 15 creatine phosphate) with $1 \%$ Triton X-100 (Thermo Scientific, Waltham, Mass) and a high concentration of protease inhibitors ([in $\mathrm{mM}$ ] $0.1 \mathrm{E} 64,0.4$ Leupeptin and 0.5 PMSF) for $24-48 \mathrm{hrs}$ at $4^{\circ} \mathrm{C}$. After skinning, tissues were washed with Triton-free relaxing solution with protease inhibitors then infiltrated with a $50 \%(\mathrm{vol} / \mathrm{vol})$ solution of relaxing solution and glycerol at $4^{\circ} \mathrm{C}$ then stored at $-20^{\circ} \mathrm{C}$. Small fibers were dissected, glued to aluminum clips, and attached at either end to a force transducer (AE801, SensorOne, Sausalito, Calif) and length motor (308B, Aurora Scientific, Aurora ON, Canada) and washed with relaxing solution with inhibitors. Crosssectional area (CSA) was measured $\left(0.023 \pm 0.002 \mathrm{~mm}^{2}\right)$. All fibers activated at $2.0 \mu \mathrm{m}$ sarcomere lengths (SL) using a pCa 4.0 solution to confirm fiber quality, achieving tensions of $39.5 \pm 2.5 \mathrm{mN} / \mathrm{mm}^{2}$. Length perturbations and force measurements were performed using a custom LabVIEW interface (National Instruments, Austin, Tex)

Cardiac muscle fibers were stretched with two length perturbations to evaluate viscosity [29]. The fibers were stretched using a stretch-hold-release protocol from their slack sarcomere length to a SL of $2.15 \mu \mathrm{m}$ at 3 speeds: $0.1,1.0$, and 10.0 lengths/s. After stretch, the fibers were held at their stretched length for 90 seconds, followed by a symmetric release. A sinusoidal frequency sweep was applied at the end of hold of the 0.1 length/s stretch with frequencies from 0.1 to $400 \mathrm{~Hz}$ at an amplitude of $+/-2 \%$ of the fiber length. 10 minutes of recovery time in between stretches was used. In order to probe the dependence on sarcomeric strain, frequency sweeps were also imparted at a SL of $2.25 \mu \mathrm{m}$ (fibers were stretched from slack to a SL of $2.25 \mu \mathrm{m}$ at 0.1 lengths/sec).

To test the magnitude of PEVK-actin interactions, recombinant PEVK fragments were expressed in E. coli and purified as previously described [32]. Briefly, cDNA fragments were amplified from human cDNA with primer pairs corresponding to the human cardiac I27-PEVK-I84 fragment with the flanking Ig domains included to enhance the stability of the protein. Amplified cDNA fragments were inserted into a pETM11 vector, expressed in $E$. coli, and the obtained fragments were sequence verified. Fragments were purified then dialyzed into PBS ([in $\mathrm{mM}$ ] $\left.2.7 \mathrm{KCl}, 1.5 \mathrm{KH}_{2} \mathrm{PO}_{4}, 137 \mathrm{NaCl}, 8 \mathrm{Na}_{2} \mathrm{PO}_{4}\right)$ and $10 \%$ glycerol, aliquoted, flash frozen, and stored at $-80^{\circ} \mathrm{C}$. For use, proteins were thawed and dialyzed in relaxing solution with protein inhibitors. PEVK fragments were added to the pig fibers to a final concentration of $22 \mu \mathrm{M}$ in relaxing solution with protease inhibitors, allowed to incubate for $>1 \mathrm{hr}$, and probed with the mechanical protocol described above.

2.2. Analysis of Skinned Muscle Mechanics. Data was analyzed offline in a custom LabVIEW VI. Stress was calculated by dividing measured force by cross-sectional area. For stretchhold experiments, stress was measured at the end of the 
stretch to calculate the peak stress and again at the end of the hold ( $90 \mathrm{sec}$ after peak) to calculate a steady state stress. Analysis methodology was adapted from previous work $[33,34]$. The viscous stress is calculated as the peak minus steady-state stress during the hold. The viscosity was calculated as the slope between the viscous stress and the $\log _{10}$ of the stretch speed (in lengths/s).

Frequency sweep data was analyzed to obtain viscous moduli [35]. The stress was used to first calculate the complex stiffness as the stress divided by the amplitude of the imposed length change (normalized to fiber length). Phase delay was calculated as the phase difference between the stress and strain signals. The viscous modulus was then calculated as complex stiffness times the sine of the phase delay [35] (see inset of Figure 3(a)) at all frequencies.

2.3. Statistics. Data analysis was performed in MS Excel. Student $t$-test was used to compare the viscosity (slope and viscous modulus at a given frequency) between groups. A $P$ value $<0.05$ was considered significant.

\section{Results}

Using titin protein gels, we first established the titin isoform expression ratio of porcine cardiac LV tissues and found that the N2BA: N2B isoform ratio is $\sim 1.0$ (Figures 1 (a) and $1(\mathrm{~b}))$. This expression ratio is much higher than that of the mouse LV $(\sim 0.2)$ and is slightly higher than that of normal human LV and similar to that of human LV of DCM patients (Figure 1(b)). To quantify viscosity, we stretched skinned pig LV muscle from their slack SL ( $1.9 \mu \mathrm{m})$ to $2.15 \mu \mathrm{m}$ and then held length constant for 90 sec to observe stress relaxation, followed by a release back to the slack length. Figure 1(c) shows two examples of such stretch-hold-release experiments, one carried out in relaxing solution (Ctrl) and the other in relaxing solution to which exogenous PEVK had been added (we confirmed the purity of the expressed PEVK using gels, see inset of Figure $1(\mathrm{c})$ ). We measured viscous stress $\left(\sigma_{v}\right)$ from the stress relaxation during the hold phase of the protocol (explained at top of Figure 2(a)). Viscous stress was measured at three stretch speeds encompassing subphysiologic (0.1 lengths/s) to supraphysiologic speeds (10 lengths/s) and the magnitude of the stress relaxation, defined by the peak minus steadystate stress (Figure 2(a), inset), was determined at each speed. Viscosity was quantified as the slope of stress relaxation versus (log) speed. At SL of $2.15 \mu \mathrm{m}$, the viscosity (slope) was reduced by $35 \%(P=0.03)$ when fibers were incubated with recombinant PEVK (Figure 2(b)). These results indicate that PEVK-actin interactions are present and relevant in tissues expressing high levels of the compliant titin N2BA isoform.

We also measured the viscous moduli during a sinusoidal oscillation protocol at frequencies from 0.1 to $400 \mathrm{~Hz}$ and using an amplitude of $2 \%$ of the fiber length and repeated the protocol in the absence and presence of recombinant PEVK. Frequency analysis provides a robust method to quantify the viscous response of a tissue at a wide array of speeds
$[29,35]$. To quantify the modulus, the ratio of the stress response and length perturbation was first used to calculate a complex stiffness and the phase delay between stress and length is used to calculate the viscous modulus (Figure 3(a)). The viscous modulus was reduced by $40 \%$ by the treatment with recombinant PEVK at SL of $2.15 \mu \mathrm{m}$ (Figure 3(b)). We also performed experiments at a SL of $2.25 \mu \mathrm{m}$ and found that the viscous moduli were reduced by $50 \%$ at all frequencies (Figure 3(c)). Importantly, the viscous modulus maintained a constant reduction at physiologic frequencies ( $\sim 0.5-2 \mathrm{~Hz}$ corresponding to the heart rate in the pig) for both SL ranges. These data indicate that the introduction of recombinant PEVK does reduce the viscosity of the system at all frequencies, including frequencies corresponding to physiologic heart rates, and reveals that it does this in a SLdependent manner.

\section{Discussion}

In resisting the rapid expansion of the left ventricle, viscosity is an important modulator of diastolic function $[2,5-7]$. While previous work has shown a contribution of PEVKactin interactions to viscosity both in vitro $[13,26,36$, 37] and in vivo [29], such findings were largely obtained in small rodents that express titin that is stiffer than in larger mammals and often at speeds below $[13,36]$ or above [26] physiologic rates. Yet the relevance to human cardiac function and dysfunction is not direct, owing to the increased expression of the compliant N2BA isoform that contains additional Ig domains and a longer PEVK domain. To determine the viscous properties of titin in a large animal model with a human-like titin isoform expression ratio, we investigated pig myocardial tissues. Additionally, we utilized recombinant PEVK proteins to interrupt native PEVK-actin interactions.

PEVK-actin interactions in the pig were significantly reduced by competitive binding of recombinant PEVK to the actin filament. This reduced the viscous response to stretch by $35 \%$ and the viscous moduli by more than $40 \%$ at a SL of $2.15 \mu \mathrm{m}$ (Figures 2 and 3). At longer sarcomere lengths $(2.25 \mu \mathrm{m})$ that are likely to be reached during diastole in pigs [38], the viscous moduli obtained with the sinusoidal analysis increased to $\sim 50 \%$. Thus, PEVK-actin interaction is a major source of viscosity in large mammals like the pig. These novel findings are the first to quantify viscosity across physiologic speeds in tissues expressing high levels of compliant N2BA isoforms.

Our findings indicate that PEVK-actin interaction is likely to be physiologically important in humans where viscosity influences relaxation $[2,5,6]$. The characterization of delayed relaxation [39] has long been a clinical indicator of diastolic dysfunction in echocardiography. Recent advances in modeling further support that the delayed relaxation phenotype is caused by a viscous force [2]. Recently, we used transmitral Doppler echocardiography in a KO mouse deficient in PEVK and found a significant reduction in viscosity during early rapid filling [29]. The ability to translate the echocardiographic findings in mice to humans could not be fully appreciated until the present results on 


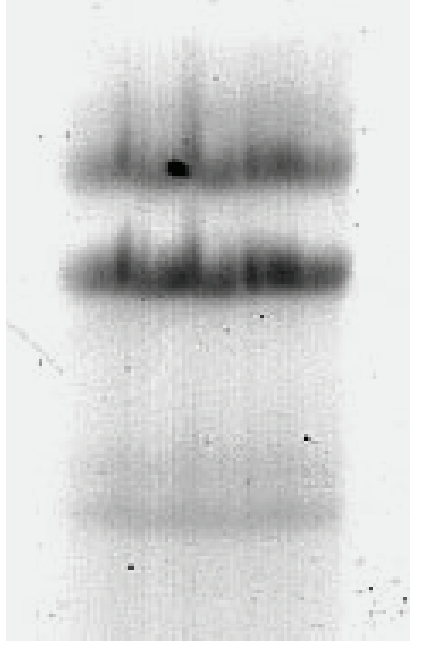

(a)

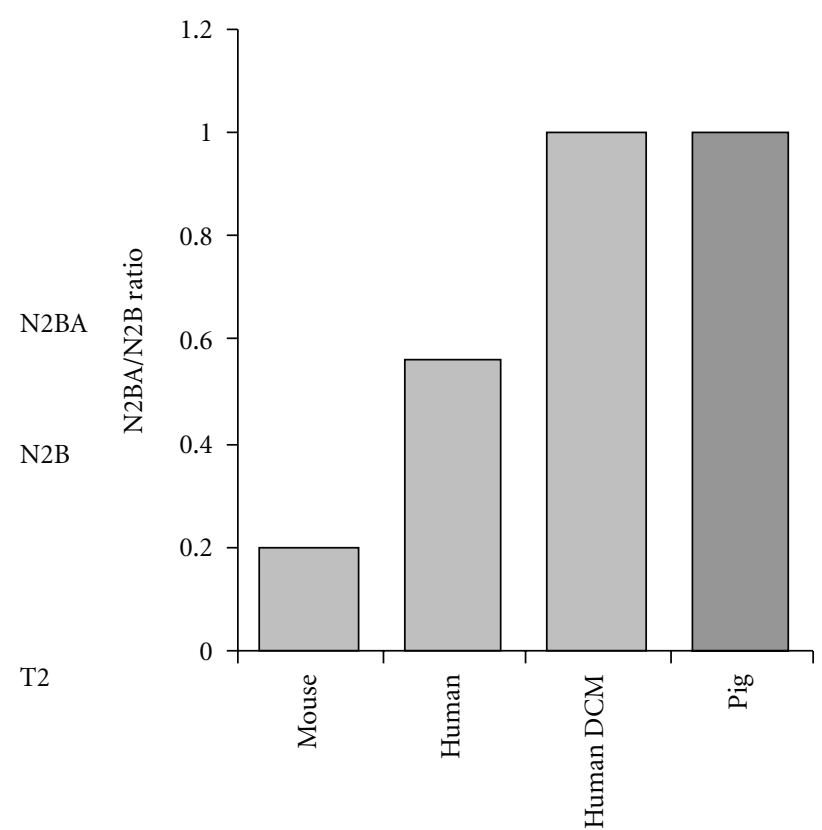

(b)
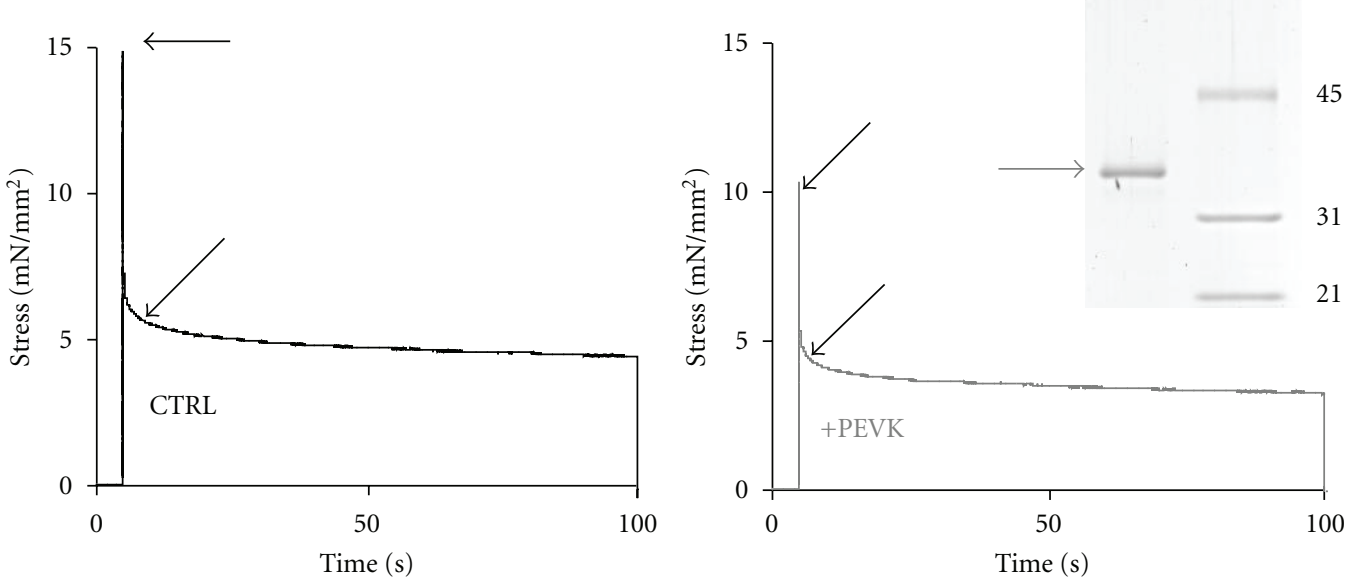

(c)

Figure 1: Porcine myocardial characteristics. (a) Pig LV tissues electrophoresed on a large pore 1\% agarose gel indicate a nearly $1: 1$ $\mathrm{N} 2 \mathrm{BA}$ : N2B ratio. (b) N2BA/N2B ratios range from 0.2 in small animals to 1.0 in human dilated cardiomyopathy (DCM). (c) Example mechanical perturbation of pig tissue from slack SL to $2.15 \mu \mathrm{m}$ at 10.0 lengths/s in control conditions and incubated with recombinant PEVK protein to inhibit native PEVK-actin interactions showing reduced stress relaxation (arrows). Inset: SDS-PAGE indicating the high purity of the recombinant PEVK protein fraction that was used. See text for details.

pig myocardium that showed a clear presence of viscosity in tissues with a high N2BA:N2B ratio. Our work establishes that despite the longer PEVK element and distinct sequence composition of the N2BA PEVK, PEVK-actin interactions are present in myocardial tissues with human-like titin isoform expression. Thus, this interaction is conserved across species, supporting the importance of these interactions to diastolic function.
The SL-dependence of the viscous modulus (Figure 3) was unexpected and may indicate that at longer SL where the PEVK is stretched to a higher degree additional binding site for actin are available than at short SL or, alternatively, that the lattice compression that occurs at long SL enhances the interaction [29]. The increased viscosity at longer SL might reduce any potential SL "overshoot" during filling $[36,40]$ and may allow for more precise end-diastolic SL control. 


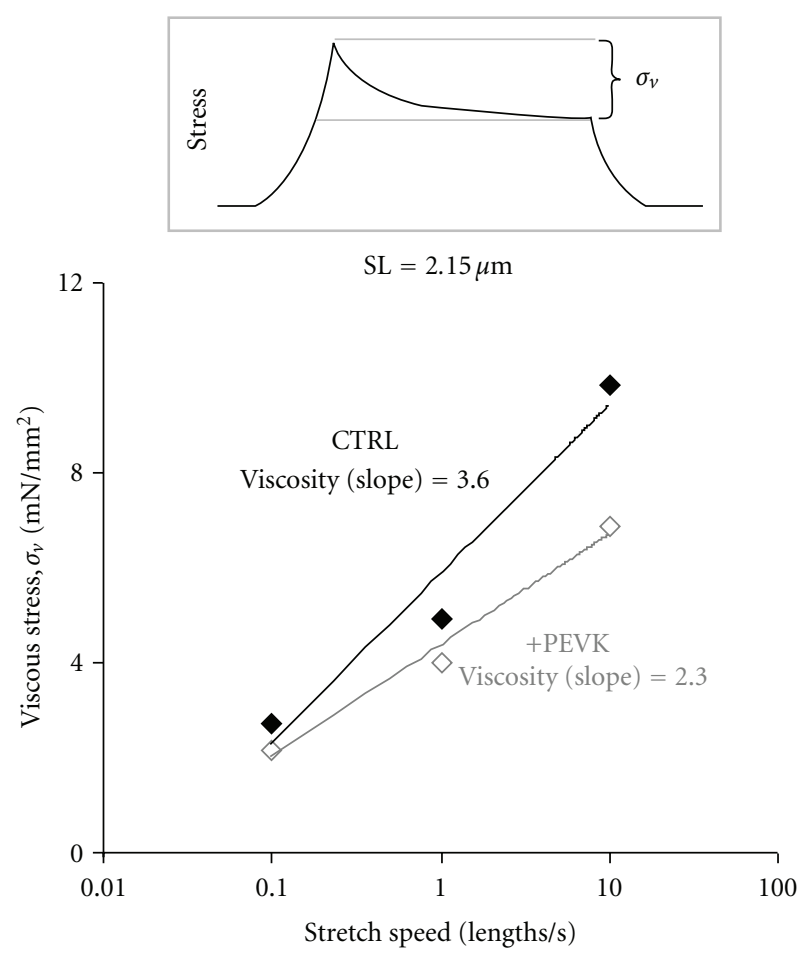

(a)

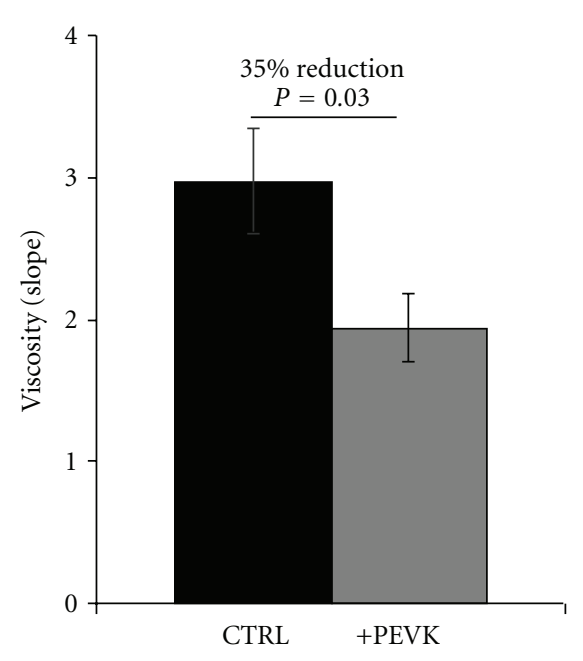

(b)

FIgURE 2: Measurement of viscosity from stress relaxation data in pig tissue. (a) Example viscosity data derived from stress relaxation in a fiber untreated (CTRL, black) and in presence of recombinant PEVK protein (+PEVK, gray). Inset: schematic of explaining how viscous stress $\left(\sigma_{v}\right)$ was determined. (b) Exogenously added PEVK reduces the viscosity (slope) by $35 \%$. See text for details.

The competition assays with recombinant PEVK reduced viscosity by only $40-50 \%$. This incomplete inhibition is possibly due to the inability of the added PEVK to compete off all native PEVK-actin interactions. Alternatively, it indicates that there is an additional source of viscosity, a possibility that is consistent with results of the PEVK KO mouse model that also show that in absence of the PEVK element myocardial tissue continues to display $\sim 50 \%$ of the viscosity found in wildtype myocardium [29]. Stretch-hold and frequency sweep protocols in myofilament extracted mouse fibers have negligible viscosity suggesting that the extracellular matrix contributes little to viscosity of the myocardium [29]. Thus, the non-PEVK source of viscosity is likely to arise from interactions that involve the myofilaments. In our studies, in PEVK KO mice, we evaluated microtubules, a known source of viscosity under pathological conditions [34, 41, 42]. However, colchicine treatment produced no effect on viscosity of the intact heart neither in WT nor in PEVK KO mice [29], suggesting that, in normal hearts, there is little contribution of microtubules to viscosity. Thin-thick filament interaction is unlikely because this predicts that viscosity would be increased when the myofilament lattice is compressed in PEVK KO tissue and this is not what we observed in our previous studies [29]. Possibilities that require future testing are intermolecular interactions between adjacent titin molecules and interactions between intermediate filaments and any of the myofilament types. In summary, although additional sources of viscosity are likely to exist, the PEVK-actin interaction is the only well-established molecular source of viscosity in cardiac tissues and contributes up to half of the total viscosity of skinned myocardium in relaxing solution.

It has previously been shown that PEVK-actin interaction can be regulated by S100A1, a calcium binding protein present in cardiac tissues (for recent review see [43]). S100A1 is a member of the S100 calcium binding proteins that is preferentially found in cardiac tissues. S100A1 normally targets to calcium handling proteins (the sarcoplasmic reticulum, RyR2), but also localizes in the Iband of the sarcomere [13]. When present in the sarcomere and activated by the presence of physiological calcium levels, S100A1 inhibits PEVK-actin interactions [13, 36]. Thus, PEVK-actin interaction-based viscosity can be turned off during systole when high calcium levels are present in the cytosol and turned on again during diastole when calcium levels fall. A second mechanism shown to influence PEVKactin interactions is protein kinase C-alpha (PKC $\alpha$ ) phosphorylation of titin. PKC $\alpha$ phosphorylation occurs at two sites in the PEVK element that are conserved across a large number of mammalian species including the mouse, pig, and human [32]. In both pig and mouse skinned cardiac fibers $[32,44], \mathrm{PKC} \alpha$ treatment increased viscosity by $20-30 \%$ at physiologic speeds; the effect was amplified by pretreating 

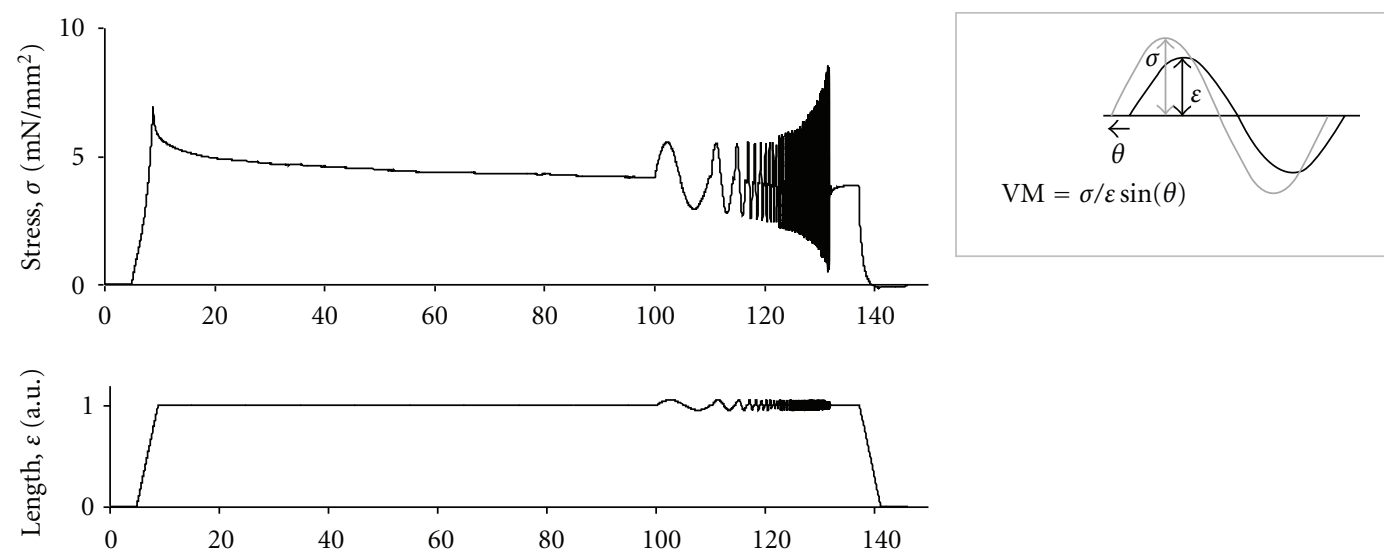

(a)
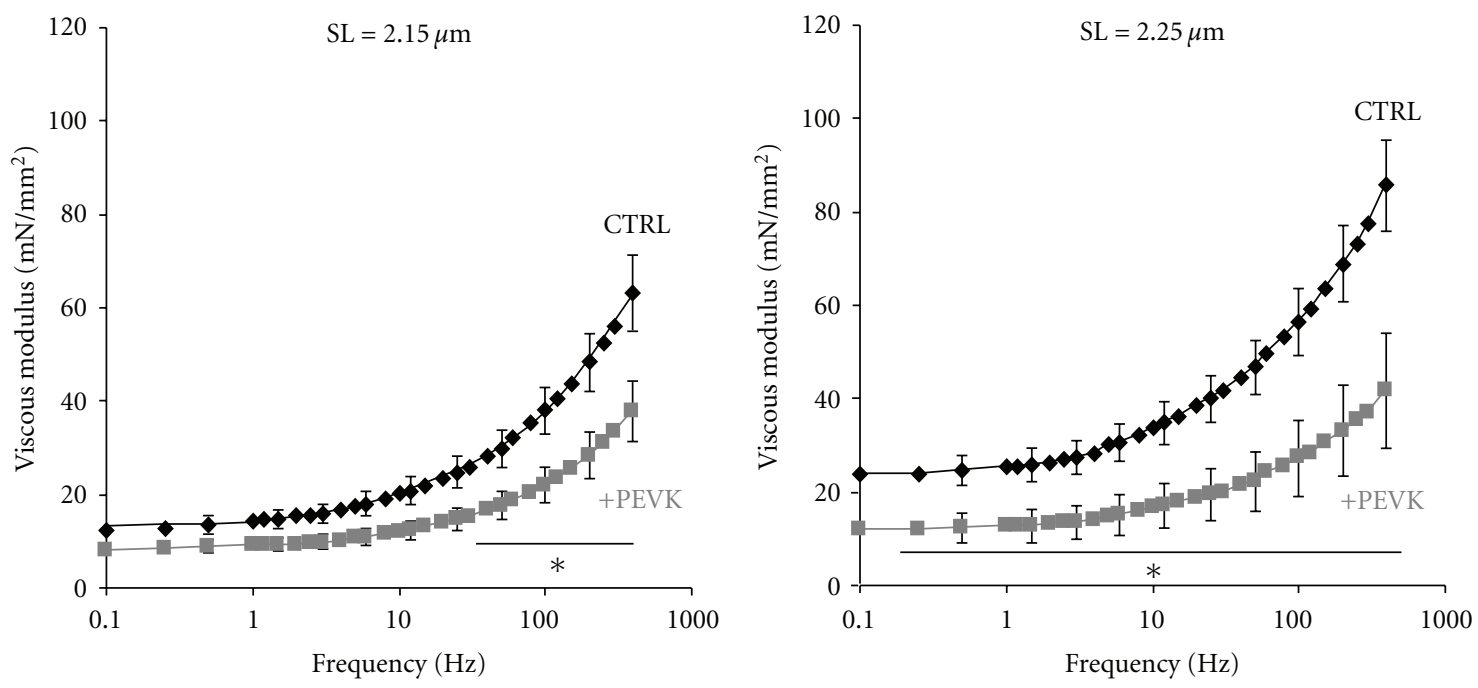

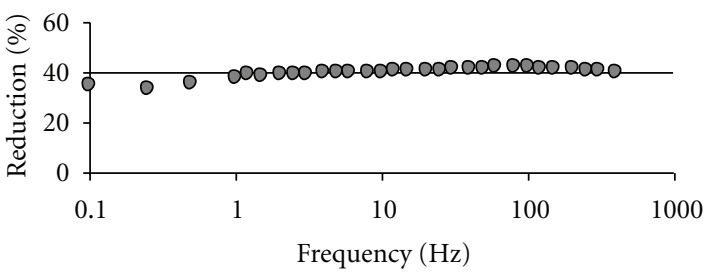

(b)

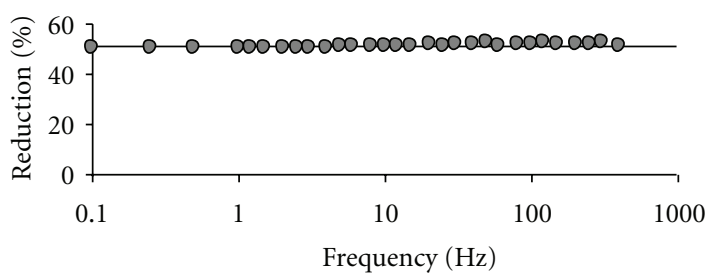

(c)

FIGURE 3: Measurement of viscous moduli via sinusoidal analysis in pig tissue. (a) The frequency sweep perturbation is imparted after a 90-second hold at SL of 2.15 or $2.25 \mu \mathrm{m}$. The viscous modulus is calculated utilizing the magnitude of the stress response, magnitude of the length perturbation and phase delay (schematic, right). (b) The viscous modulus measured in control tissues at SL of $2.15 \mu \mathrm{m}$ (CTRL, black) is reduced in the presence of recombinant PEVK proteins (+PEVK, gray) by $40 \%$. (c) At a SL of $2.25 \mu \mathrm{m}$, adding PEVK protein ( + PEVK, gray) reduces the viscous modulus at all frequencies. ${ }^{*}$ indicates $P<0.05$ across the frequencies noted by the horizontal bar. See text for details.

the fibers by protein phosphatase 1 (PP1) to dephosphorylate titin [32]. These changes in viscosity indicate a potentially large dynamic range by which viscosity can be modulated. The understanding of the functional effects of PKC $\alpha$ phosphorylation and its regulation may aid in our understanding of the underlying dysfunction, for example, in heart failure where PKC $\alpha$ is upregulated $[45,46]$. These factors provide a potential path for clinical management of cardiac diastolic dysfunction.

\section{Conclusion}

This study establishes the presence of PEVK-actin interactions in a large mammal, the pig, and is the first to conclusively determine with physiological stretch protocols the magnitude of PEVK-actin interactions in hearts with a human-like titin isoform expression ratio. Competition assays with exogenous recombinant protein show that the magnitude of this contribution is up to $50 \%$ of the total 
viscosity seen in these tissues. Furthermore, we found that PEVK-based viscosity is larger at longer SL, a property that might aid in preventing SL overshoot at the end of filling, and because of the influence of PEVK-actin interactions on early rapid filling, modulation of these interactions are of potential clinical importance.

\section{Acknowledgments}

The authors gratefully acknowledge the assistance of Luann Wyly for expert animal care and other lab members for advice and assistance. This work was supported by grants from the American Heart Association (0825748G) and the NIH (T32 HL07249-31) to C. S. Chung; by the Deutsche Forschungsgemeinschaft to S. Labeit; the NIH (R01 HL062881) and a generous gift from Alan and Alfie Norville to H. L. Granzier.

\section{References}

[1] M. Ohno, C. P. Cheng, and W. C. Little, "Mechanism of altered patterns of left ventricular filling during the development of congestive heart failure," Circulation, vol. 89, no. 5, pp. 22412250, 1994.

[2] L. Shmuylovich and S. J. Kovács, "E-wave deceleration time may not provide an accurate determination of LV chamber stiffness if LV relaxation/viscoelasticity is unknown," American Journal of Physiology - Heart and Circulatory Physiology, vol. 292, no. 6, pp. H2712-H2720, 2007.

[3] M. R. Zile and D. L. Brutsaert, "New concepts in diastolic dysfunction and diastolic heart failure: part I: diagnosis, prognosis, and measurements of diastolic function," Circulation, vol. 105, no. 11, pp. 1387-1393, 2002.

[4] C. S. Chung and H. L. Granzier, "Contribution of titin and extracellular matrix to passive pressure and measurement of sarcomere length in the mouse left ventricle," Journal of Molecular and Cellular Cardiology, vol. 50, no. 4, pp. 731-739, 2011.

[5] C. S. Chung and S. J. Kovács, "Physical determinants of left ventricular isovolumic pressure decline: model prediction with in vivo validation," American Journal of PhysiologyHeart and Circulatory Physiology, vol. 294, no. 4, pp. H1589H1596, 2008.

[6] D. A. Kass, J. G. F. Bronzwaer, and W. J. Paulus, "What mechanisms underlie diastolic dysfunction in heart failure?" Circulation Research, vol. 94, no. 12, pp. 1533-1542, 2004.

[7] S. J. Kovács, B. Barzilai, and J. E. Pérez, "Evaluation of diastolic function with Doppler echocardiography: the PDF formalism," American Journal of Physiology-Heart and Circulatory Physiology, vol. 252, no. 1, part 2, pp. H178-H187, 1987.

[8] K. Wang, J. McClure, and A. Tu, "Titin: major myofibrillar components of striated muscle," Proceedings of the National Academy of Sciences of the United States of America, vol. 76, no. 8, pp. 3698-3702, 1979.

[9] K. Maruyama, "Connectin, an elastic protein from myofibrils," Journal of Biochemistry, vol. 80, no. 2, pp. 405-407, 1976.

[10] M. M. Lewinter and H. Granzier, "Cardiac titin: a multifunctional giant," Circulation, vol. 121, no. 19, pp. 2137-2145, 2010.

[11] W. A. Linke and M. Krüger, "The giant protein titin as an integrator of myocyte signaling pathways," Physiology, vol. 25, no. 3, pp. 186-198, 2010.
[12] H. L. Granzier and S. Labeit, "The giant protein titin: a major player in myocardial mechanics, signaling, and disease," Circulation Research, vol. 94, no. 3, pp. 284-295, 2004.

[13] R. Yamasaki, M. Berri, Y. Wu et al., "Titin-actin interaction in mouse myocardium: passive tension modulation and its regulation by calcium/S100A1," Biophysical Journal, vol. 81, no. 4, pp. 2297-2313, 2001.

[14] H. P. Erickson, "Reversible unfolding of fibronectin type III and immunoglobulin domains provides the structural basis for stretch and elasticity of titin and fibronectin," Proceedings of the National Academy of Sciences of the United States of America, vol. 91, no. 21, pp. 10114-20118, 1994.

[15] L. Tskhovrebova, J. Trinick, J. A. Sleep, and R. M. Simmons, "Elasticity and unfolding of single molecules of the giant muscle protein titin,” Nature, vol. 387, no. 6630, pp. 308-312, 1997.

[16] M. Rief, M. Gautel, A. Schemmel, and H. E. Gaub, "The mechanical stability of immunoglobulin and fibronectin III domains in the muscle protein titin measured by atomic force microscopy," Biophysical Journal, vol. 75, no. 6, pp. 3008-3014, 1998.

[17] M. Gautel, E. Lehtonen, and F. Pietruschka, "Assembly of the cardiac I-band region of titin/connectin: expression of the cardiac-specific regions and their structural relation to the elastic segments," Journal of Muscle Research and Cell Motility, vol. 17, no. 4, pp. 449-461, 1996.

[18] K. Trombitás, M. Greaser, S. Labeit et al., "Titin extensibility in situ: entropic elasticity of permanently folded and permanently unfolded molecular segments," Journal of Cell Biology, vol. 140, no. 4, pp. 853-859, 1998.

[19] K. Trombitás, Y. Wu, M. McNabb et al., "Molecular basis of passive stress relaxation in human soleus fibers: assessment of the role of immunoglobulin-like domain unfolding," Biophysical Journal, vol. 85, no. 5, pp. 3142-3153, 2003.

[20] M. Helmes, K. Trombitás, T. Centner et al., "Mechanically driven contour-length adjustment in rat cardiac titin's unique N2B sequence: titin is an adjustable spring," Circulation Research, vol. 84, no. 11, pp. 1339-1352, 1999.

[21] H. Granzier, M. Kellermayer, M. Helmes, and K. Trombitás, "Titin elasticity and mechanism of passive force development in rat cardiac myocytes probed by thin-filament extraction," Biophysical Journal, vol. 73, no. 4, pp. 2043-2053, 1997.

[22] T. Funatsu, E. Kono, H. Higuchi et al., "Elastic filaments in situ in cardiac muscle: deep-etch replica analysis in combination with selective removal of actin and myosin filaments," Journal of Cell Biology, vol. 120, no. 3, pp. 711-724, 1993.

[23] M. S. Kellermayer and H. L. Granzier, "Calcium-dependent inhibition of in vitro thin-filament motility by native titin," FEBS Letters, vol. 380, no. 3, pp. 281-286, 1996.

[24] S. Kimura, K. Maruyama, and Y. P. Huang, "Interactions of muscle $\beta$-connectin with myosin, actin, and actomyosin at low ionic strengths," Journal of Biochemistry, vol. 96, no. 2, pp. 499-506, 1984.

[25] A. Soteriou, M. Gamage, and J. Trinick, "A survey of interactions made by the giant protein titin," Journal of Cell Science, vol. 104, no. 1, part 1, pp. 119-123, 1993.

[26] M. Kulke, S. Fujita-Becker, E. Rostkova et al., "Interaction between PEVK-titin and actin filaments origin of a viscous force component in cardiac myofibrils," Circulation Research, vol. 89, no. 10, pp. 874-881, 2001.

[27] W. A. Linke, M. Ivemeyer, S. Labeit, H. Hinssen, J. C. Rüegg, and M. Gautel, "Actin-titin interaction in cardiac myofibrils: probing a physiological role," Biophysical Journal, vol. 73, no. 2, pp. 905-919, 1997. 
[28] H. L. Granzier, M. H. Radke, J. Peng et al., "Truncation of titin's elastic PEVK region leads to cardiomyopathy with diastolic dysfunction," Circulation Research, vol. 105, no. 6, pp. 557-564, 2009.

[29] C. S. Chung, M. Methawasin, O. L. Nelson et al., "Titin based viscosity in ventricular physiology: an integrative investigation of PEVK-actin interactions," Journal of Molecular and Cellular Cardiology, vol. 51, no. 3, pp. 428-434, 2011.

[30] M. Greaser, "Identification of new repeating motifs in titin," Proteins, vol. 43, no. 2, pp. 145-149, 2001.

[31] A. Nagy, P. Cacciafesta, L. Grama, A. Kengyel, A. MálnásiCsizmadia, and M. S. Kellermayer, "Differential actin binding along the PEVK domain of skeletal muscle titin," Journal of Cell Science, vol. 117, part 24, pp. 5781-5789, 2004.

[32] C. Hidalgo, B. Hudson, J. Bogomolovas et al., "PKC phosphorylation of titin's PEVK element: a novel and conserved pathway for modulating myocardial stiffness," Circulation Research, vol. 105, no. 7, pp. 631-638, 2009.

[33] P. P. de Tombe and H. E. ter Keurs, "An internal viscous element limits unloaded velocity of sarcomere shortening in rat myocardium," Journal of Physiology, vol. 454, pp. 619-642, 1992.

[34] T. S. Harris, C. F. Baicu, C. H. Conrad et al., "Constitutive properties of hypertrophied myocardium: cellular contribution to changes in myocardial stiffness," American Journal of Physiology-Heart and Circulatory Physiology, vol. 282, no. 6, pp. H2173-H2182, 2002.

[35] H. L. Granzier and K. Wang, "Interplay between passive tension and strong and weak binding cross-bridges in insect indirect flight muscle: a functional dissection by gelsolinmediated thin filament removal," Journal of General Physiology, vol. 101, no. 2, pp. 235-270, 1993.

[36] H. Fukushima, C. S. Chung, and H. Granzier, "Titin-isoform dependence of titin-actin interaction and its regulation by S100A1/ $\mathrm{Ca}^{2+}$ in skinned myocardium," Journal of Biomedicine and Biotechnology, vol. 2010, Article ID 727239, 9 pages, 2010.

[37] W. A. Linke, M. Kulke, H. Li et al., "PEVK domain of titin: an entropic spring with actin-binding properties," Journal of Structural Biology, vol. 137, no. 1-2, pp. 194-205, 2002.

[38] M. M. Lewinter, J. Popper, M. McNabb, L. Nyland, S. B. Bell, and H. Granzier, "Extensible behavior of titin in the miniswine left ventricle," Circulation, vol. 121, no. 6, pp. 768-774, 2010.

[39] S. F. Nagueh, C. P. Appleton, T. C. Gillebert et al., "Recommendations for the evaluation of left ventricular diastolic function by echocardiography," Journal of the American Society of Echocardiography, vol. 22, no. 2, pp. 107-133, 2009.

[40] L. Shmuylovich, C. S. Chung, and S. J. Kovács, "Point: left ventricular volume during diastasis is the physiological in vivo equilibrium volume and is related to diastolic suction," Journal of Applied Physiology, vol. 109, no. 2, pp. 606-608, 2010.

[41] J. D. Stroud, C. F. Baicu, M. A. Barnes, F. G. Spinale, and M. R. Zile, "Viscoelastic properties of pressure overload hypertrophied myocardium: effect of serine protease treatment," American Journal of Physiology-Heart and Circulatory Physiology, vol. 282, no. 6, pp. H2324-H2335, 2002.

[42] S. Nishimura, S. Nagai, M. Katoh et al., "Microtubules modulate the stiffness of cardiomyocytes against shear stress," Circulation Research, vol. 98, no. 1, pp. 81-87, 2006.

[43] M. Vlkers, D. Rohde, C. Goodman, and P. Most, "S100A1: a regulator of striated muscle sarcoplasmic reticulum $\mathrm{Ca}^{2+}$ handling, sarcomeric, and mitochondrial function," Journal of Biomedicine and Biotechnology, vol. 2010, Article ID 178614, 10 pages, 2010.
[44] B. D. Hudson, C. G. Hidalgo, M. Gotthardt, and H. L. Granzier, "Excision of titin's cardiac PEVK spring element abolishes PKC $\alpha$-induced increases in myocardial stiffness," Journal of Molecular and Cellular Cardiology, vol. 48, no. 5, pp. 972-978, 2010.

[45] E. Churchill, G. Budas, A. Vallentin, T. Koyanagi, and D. Mochly-Rosen, "PKC isozymes in chronic cardiac disease: possible therapeutic targets?" Annual Review of Pharmacology and Toxicology, vol. 48, pp. 569-599, 2008.

[46] S. S. Palaniyandi, L. Sun, J. C. Ferreira, and D. MochlyRosen, "Protein kinase C in heart failure: a therapeutic target?" Cardiovascular Research, vol. 82, no. 2, pp. 229-239, 2009. 

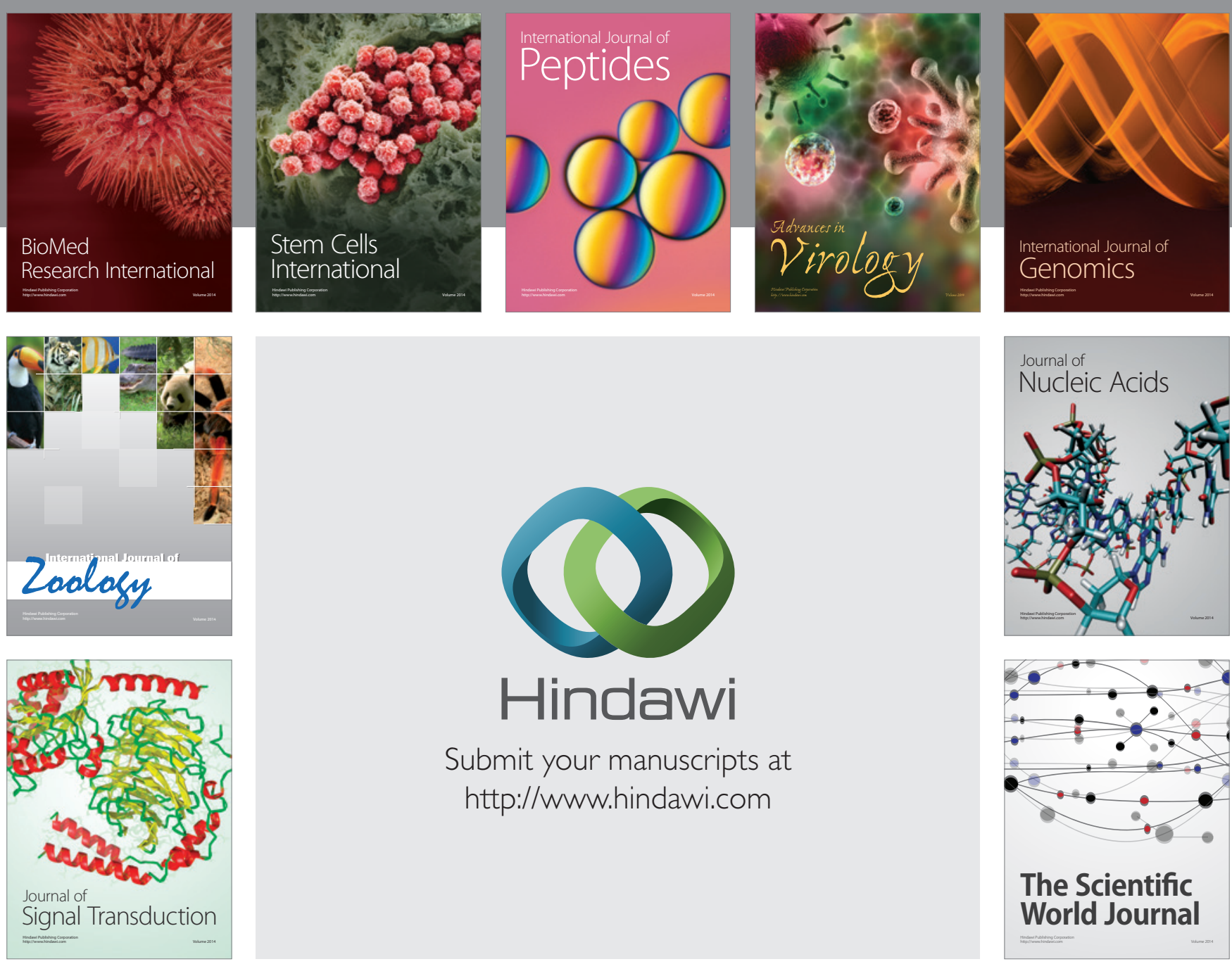

Submit your manuscripts at

http://www.hindawi.com
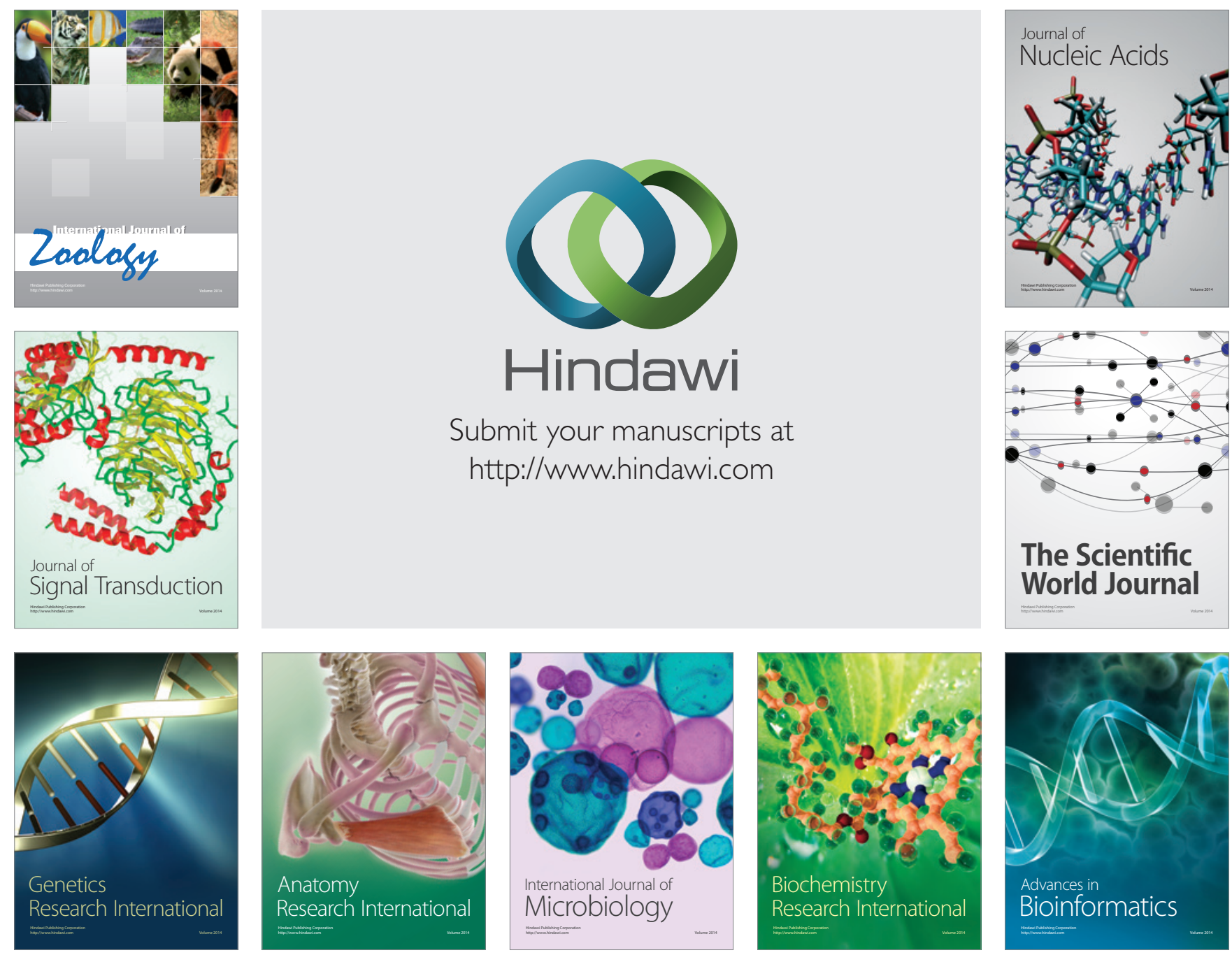

The Scientific World Journal
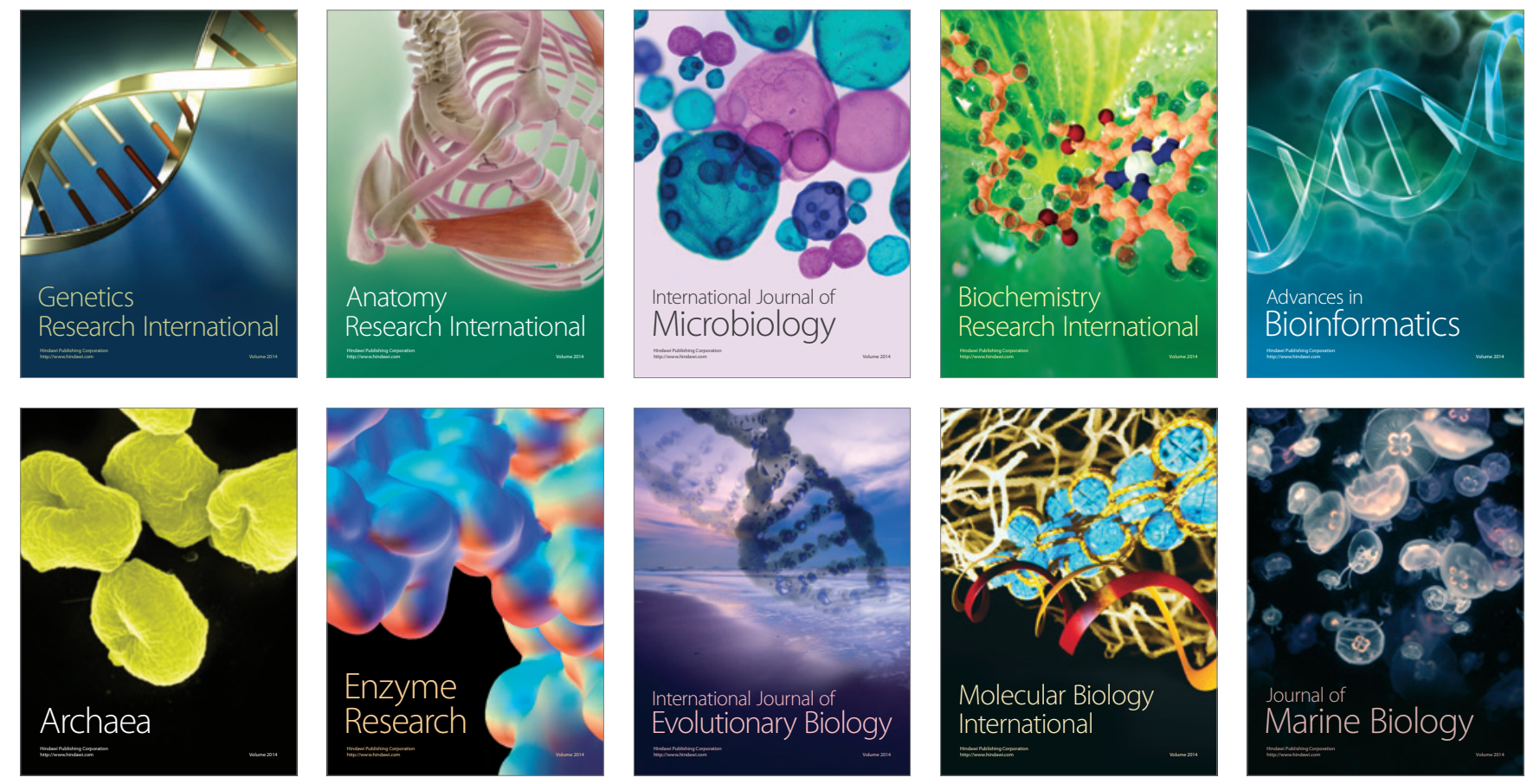